OPEN ACCESS

Edited by:

Yinduo Ji,

University of Minnesota Twin Cities,

United States

Reviewed by:

Marat R. Sadykov,

University of Nebraska Medical

Center, United States

William Schwan,

University of Wisconsin-La Crosse,

United States

${ }^{*}$ Correspondence:

Zihao Teng

tengzh7@163.com

Jianfeng Wang

wjf927@jlu.edu.cn

Specialty section:

This article was submitted to

Molecular Bacterial Pathogenesis,

a section of the journal

Frontiers in Cellular and Infection

Microbiology

Received: 01 March 2019

Accepted: 26 April 2019

Published: 15 May 2019

Citation:

Jiang L, Yi T, Shen Z, Teng $Z$ and

Wang J (2019) Aloe-emodin Attenuates Staphylococcus aureus Pathogenicity by Interfering With the

Oligomerization of $\alpha$-Toxin.

Front. Cell. Infect. Microbiol. 9:157.

doi: 10.3389/fcimb.2019.00157

\section{Aloe-emodin Attenuates Staphylococcus aureus Pathogenicity by Interfering With the Oligomerization of $\alpha$-Toxin}

\author{
Lanxiang Jiang ${ }^{1}$, Tian $Y^{2}{ }^{2}$, Ziying Shen ${ }^{3}$, Zihao Teng ${ }^{1 *}$ and Jianfeng Wang ${ }^{1,2 *}$ \\ ${ }^{1}$ Department of Dermatology, Second Hospital of Jilin University, Jilin University, Changchun, China, ${ }^{2}$ Key Laboratory of \\ Zoonosis Research, Ministry of Education, Institute of Zoonosis, College of Animal Science, Jilin University, Changchun, \\ China, ${ }^{3}$ Laboratory Animal Center, College of Animal Sciences, Jilin University, Changchun, China
}

$\alpha$-toxin, an essential virulence factor secreted by Staphylococcus aureus (S. aureus), is a critical exotoxin in multiple infections. In this study, we found that aloe-emodin $(A E)$, a natural compound lacking anti-S. aureus activity, could inhibit the hemolytic activity of $\alpha$-toxin. Oligomerization assays, molecular dynamics simulations, and fluorescence-quenching analyses were used to determine the mechanism of this inhibition. The oligomerization of $\alpha$-toxin was restricted by the engagement of $A E$ with $\mathrm{K} 110, \mathrm{~T} 112$, and $\mathrm{M} 113$ of the toxin, which eventually resulted in inhibition of the hemolytic activity. Lactate dehydrogenase and live/dead assays demonstrated that AE decreased the injury of human lung epithelial cells (A549) and mouse lung macrophages (MH-S) mediated by $S$. aureus. Furthermore, treatment with $A E$ showed robust protective effects in mice infected by $S$. aureus. These findings suggest that $A E$ effectively inhibited the pore-forming activity of $\alpha$-toxin and showed a protective effect against $S$. aureus virulence in vitro and in vivo, which may provide a new strategy and new antibacterial agent for clinical treatment of $S$. aureus infections.

Keywords: Staphylococcus aureus, $\alpha$-toxin, aloe-emodin, antibiotic-resistant, pneumonia

\section{INTRODUCTION}

Staphylococcus aureus (S. aureus), one of the most common pathogens, is a critical cause of many local and systemic infections ranging from pneumonia, sepsis, and andocarditis, to osteomyelitis. Among these diseases, $S$. aureus pneumonia is one of the most serious ventilator-associated infections, with $\sim 10-25 \%$ mortality and, for some secondary infections, the rate can even reach 75\% (Gillet et al., 2002; Del Giudice et al., 2011; Li et al., 2011; Chastre et al., 2014). Although lactams, aminoglycosides, tetracyclines, sulfonamides, and other major antimicrobial drugs have been commonly used in the past century, we still cannot effectively inhibit the S. aureus pneumonia observed in the clinic most of the time. However, the abuse of antibiotics has led to many resistant strains, and treatment of $S$. aureus infections has cost at least 450 million dollars due to the increasing resistance (Parvizi et al., 2010; Song et al., 2010). In Europe, $\sim 10-25 \%$ of $S$. aureus isolated from hospitals were observed to be methicillin-resistant S. aureus (MRSA), and the proportion has reached 50\% in some regions (Commun, 2011). Even worse, the proportion of MRSA appeared to reach the highest level in years in parts of east Asia, such as Taiwan and South Korea, with an average rate of 77.6\% (Chen and Huang, 2014). Since the twentieth century, 
the multiresistance of MRSA has become more complicated, which typically results in a delay in clinical treatment (Mendes et al., 2013). Currently, vancomycin is the most commonly used drug to treat MRSA-associated pneumonia (Wunderink et al., 2003). However, the sensitivity of MRSA to vancomycin has been gradually decreasing for years and, given the current trends, the time required for the spread of resistant strains is much less than the time required for research and application of a new medicine. Accordingly, no treatments may be available for MRSA pneumonia in the future, and we need a new treatment strategy to replace the old antibiotic use regimens. Several studies have reported that targeting virulence factors typically results in weak pathogenicity of pathogens, suggesting that this may be a promising strategy in the treatment of S. aureus pneumonia (Qiu et al., 2012a,b; Wang et al., 2016).

During infection, a variety of virulence factors are secreted for invasion and colonization, including exotoxin and surfaceassociated protein (Vandenesch et al., 2012). $\alpha$-toxin is one of the most important exotoxins produced by $S$. aureus and plays a key role in the course of multiple diseases as a pore-forming protein. It is a $33.2 \mathrm{kDa}$ water-soluble monomer encoded by hla and can oligomerize into a $232.4 \mathrm{kDa}$ membrane-inserted heptamer that penetrates the membrane (Gouaux, 1998; Nguyen and Kamio, 2004). The oligomer comprises seven monomers and consists of three major domains, including the cap domain, the rim domain, and the stem domain, which forms the transmembrane channel (Gouaux et al., 1994; Song et al., 1996). Many types of mammalian cells, including monocytes, erythrocytes, macrophages, and epithelial cells, are sensitive to $\alpha$-toxin (Gouaux, 1998; Nygaard et al., 2012). For $S$. aureus pneumonia, studies have reported the destructive effect of $\alpha$-toxin on the air-blood barrier, and a mutant strain lacking $\alpha$-toxin showed decreased toxicity in animal models (McElroy et al., 1999; Xu et al., 2015). Therefore, targeting $\alpha$-toxin is a promising therapeutic strategy for $S$. aureus infections, particularly MRSA pneumonia.

Aloe-emodin [AE; 1,8-dihydroxy-3-(hydroxymethyl)anthraquinone] (Figure 1A) is a common active compound derived from the leaves of Aloe vera and Rheum officinale (Dutta et al., 2007) that has been reported to possess antimicrobial, antiviral, and hepatoprotective activities (Eshun and He, 2004) as well as anticancer activity toward hepatoma cells, lung squamous cell carcinoma, and neuroectodemal tumors (Pecere et al., 2000; Lee, 2001; Kuo et al., 2002). In this study, we observed that $\mathrm{AE}$ can inhibit the hemolytic activity of $S$. aureus without decreasing the expression of $\alpha$-toxin. In addition, we evaluated the protective effect of AE against MRSA in vitro and in vivo.

\section{MATERIALS AND METHODS}

\section{Bacterial Strain and Reagents}

The MRSA strain USA300 (BAA-1717) used in this study was purchased from the American Type Culture Collection (ATCC). AE was purchased from Chengdu Herbpurify Co., Ltd. (purity $>98 \%$ ) (Chengdu, China) and was prepared in DMSO (Sigma-Aldrich). The bacteria were cultured in tryptic soy broth (TSB, Qingdao Hope Biol-Technology Co., Ltd.) at $37^{\circ} \mathrm{C}$.

\section{MIC Determination}

The MIC of AE against USA300 was assessed via the broth microdilution method according to Clinical and Laboratory Standards Institute guidelines. The MIC value was defined as the lowest concentration of $\mathrm{AE}$ at which no visible growth of the microorganism could be observed.

\section{Growth Curve Assay}

The USA300 strain was grown in TSB with shaking at $200 \mathrm{rpm}$ at $37^{\circ} \mathrm{C}$ to an $\mathrm{OD}$ value of 0.3 at $600 \mathrm{~nm}$, after which the cultures were aliquoted into five Erlenmeyer flasks. Various doses of AE were added into four of the cultures at concentrations of 2, 4, 8 , and $16 \mu \mathrm{g} / \mathrm{ml}$, maintaining the final concentration of DMSO in all the cultures at $1 \%(\mathrm{v} / \mathrm{v})$. The control cultures were treated with $1 \% \mathrm{DMSO}$. Following the addition of AE, the bacteria were grown at $37^{\circ} \mathrm{C}$ with shaking at $200 \mathrm{rpm}$, and the $\mathrm{OD}_{600}$ values of the cultures were monitored every $60 \mathrm{~min}$.

\section{Hemolysis Assay}

Hemolysis assays were used to determine the inhibitory effect of $\mathrm{AE}$ on the hemolytic activity of $\alpha$-toxin. The USA300 supernatants were harvested by centrifugation at $10,000 \mathrm{rpm}$ for $1 \mathrm{~min}$ at $4^{\circ} \mathrm{C}$, and then $0.1 \mathrm{ml}$ of each supernatant was added to $1 \mathrm{ml}$ of PBS buffer. The mixture was preincubated with $\mathrm{AE}$ at concentrations of $2,4,8$, and $16 \mu \mathrm{g} / \mathrm{ml}$ at $37^{\circ} \mathrm{C}$ for $20 \mathrm{~min}$. Next, $25 \mu \mathrm{l}$ of rabbit erythrocytes $\left(5 \times 10^{6} \mathrm{cells} / \mathrm{ml}\right)$ was added to each tube. Following an additional $30 \mathrm{~min}$ incubation at $37^{\circ} \mathrm{C}$, the unlysed erythrocytes were removed by centrifugation at $6,000 \mathrm{rpm}$ for $1 \mathrm{~min}$, and the hemolytic activity was assessed by determining the $\mathrm{OD}_{543}$ values of the supernatant. For the control group, $25 \mu$ l of rabbit erythrocytes was added to $975 \mu$ l distilled water, and the supernatant served as the $100 \%$ hemolysis control. The \% hemolysis of the AE-treated samples were calculated by comparing the $\mathrm{OD}_{543}$ values of the samples with that of the control cultures.

We also compared the effect of AE on the wild-type and mutant forms of $\alpha$-toxin. Purified proteins $(100 \mathrm{ng} / \mathrm{ml})$ were preincubated with various concentrations of $\mathrm{AE}$ at $37^{\circ} \mathrm{C}$ for $20 \mathrm{~min}$. Next, rabbit erythrocytes were added to the mixtures and incubated for another $30 \mathrm{~min}$ at $37^{\circ} \mathrm{C}$. Erythrocyte lysis was determined as described above.

\section{Western Blot Assay}

For the Western blot assay, USA300 was grown in TSB at $37^{\circ} \mathrm{C}$ with shaking at $200 \mathrm{rpm}$ to an $\mathrm{OD}_{600}$ of 0.3 . Next, the cultures were equally divided into five 50-ml Erlenmeyer flasks with or without $\mathrm{AE}$ at concentrations of $2,4,8$, and $16 \mu \mathrm{g} / \mathrm{ml}$ and then were cultured with shaking at $37^{\circ} \mathrm{C}$ until reaching the postexponential growth phase $\left(\mathrm{OD}_{600}\right.$ of 2.5$)$. Subsequently, the bacterial cultures were centrifuged at $10,000 \mathrm{rpm}$ for $1 \mathrm{~min}$, and the supernatants were boiled in Laemmli sample buffer and loaded on a $12 \%$ sodium dodecyl sulfate-polyacrylamide gel. The proteins were then transferred to polyvinylidene fluoride (PVDF) membranes (Wako Pure Chemical Industries, Ltd., Osaka, Japan). The membranes were blocked with $5 \%$ bovine serum albumin (Wako) in PBS at $4^{\circ} \mathrm{C}$ overnight. Next, a rabbit antibody against $\alpha$-toxin (diluted to 1:8,000; Sigma-Aldrich) was incubated 
A<smiles>O=C1c2cccc(O)c2C(=O)c2c(O)cc(CO)cc21</smiles>

C

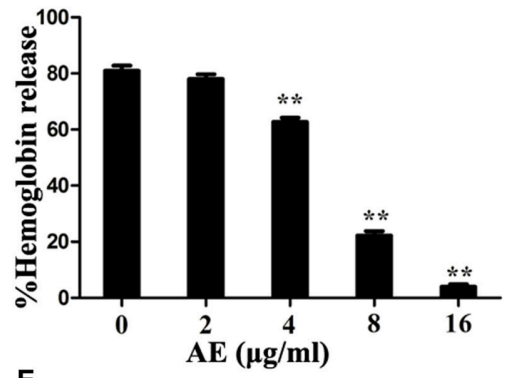

E

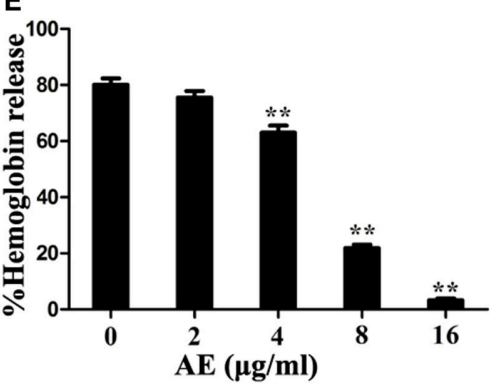

B

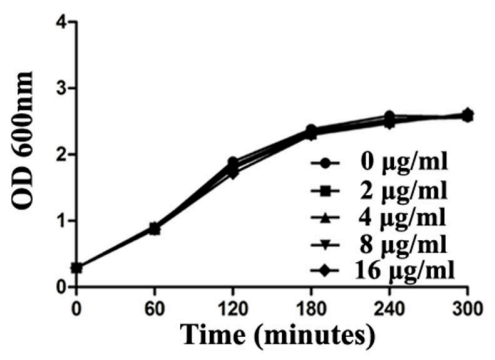

D

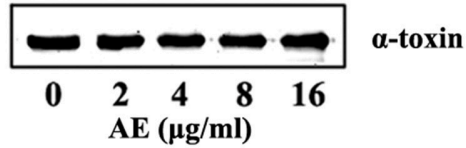

FIGURE 1 | Aloe-emodin (AE) inhibits the hemolytic activity of $\alpha$-toxin. (A) Chemical structure of AE. (B) Growth curves of USA300 treated with the indicated concentrations of $\mathrm{AE}$. The $\mathrm{OD}_{600}$ values of each sample were monitored every $60 \mathrm{~min}$. (C) USA300 was cultured with various concentrations of AE and, then, the supernatants were used for the determination of hemolytic activity. The hemolytic activity of culture supernatant of $S$. aureus co-cultued with AE was inhibited, with activities of $80.97,78.00,62.75,22.19$, and 4.00\% observed in supernatants containing 0, 2, 4, 8, and $16 \mu \mathrm{g} / \mathrm{ml}$ AE, respectively. (D) USA300 was cultured with various concentrations of $\mathrm{AE}$ and the expression of $\alpha$-toxin in the culture supernatant demonstrated by Western blot. (E) Hemolytic activity of purified $\alpha$-toxin treated with or without $\mathrm{AE}$. Bars represent the mean values of the experiments. The AE treatment reduced the observed hemolytic activity from $80.15 \%(0 \mu \mathrm{g} / \mathrm{ml})$ to 75.51 , 63.06, 22.88, and 3.26\% when supernatants contained 2, 4, 8, and $16 \mu \mathrm{g} / \mathrm{ml} \mathrm{AE}$, respectively (** indicates $P<0.01$ compared with the AE-free group; two-tailed Student's $t$-test).

with membranes at $37^{\circ} \mathrm{C}$ for $2 \mathrm{~h}$, followed by an incubation with a horseradish peroxidase-conjugated secondary antibody (diluted to 1:4,000; Sigma-Aldrich) under the same conditions. The blots were detected with Amersham ECL Western blotting detection reagents (GE Healthcare, Buckinghamshire, UK) and visualized on Tanon-5200 Chemiluminescent Imaging System (Tanon Science \& Technology Co., Ltd., Shanghai, People's Republic of China).

\section{Deoxycholate-Induced Oligomerization Assay}

To assess the deoxycholate-induced oligomerization of $\alpha$-toxin, $500 \mathrm{ng}$ of purified $\alpha$-toxin was added to $5 \mathrm{mM}$ deoxycholate and incubated with $\mathrm{AE}$ at concentrations of $2,4,8$, and $16 \mu \mathrm{g} / \mathrm{ml}$ at $22^{\circ} \mathrm{C}$ for $20 \mathrm{~min}$. Then, the samples were placed in Laemmli sample buffer without $\beta$-mercaptoethanol at $55^{\circ} \mathrm{C}$ for $10 \mathrm{~min}$, and the effect of $\mathrm{AE}$ on protein oligomerization was assessed by Western blot assays as described above.

\section{Molecular Modeling}

The initial structure of $\alpha$-toxin was obtained from the 3D X-ray structure (PDB code: $4 \mathrm{YHD}$ ). To obtain the starting structure of the AE/ $\alpha$-toxin complex for MD simulation, we used a standard docking procedure for a rigid protein and a flexible ligand using AutoDock 4 (Morris et al., 2009; Hu et al., 2010). Subsequently, the MD simulation of the complex systems was performed, and the detailed processes of the computational analyses were described in a previous report (Dong et al., 2013; Niu et al., 2013).

\section{Binding Affinity Determination of Ligands With Proteins}

To investigate the importance of Lys110, Tyr112, and Met113 in the binding of $\alpha$-toxin and AE, we mutated these amino acids to 
alanine. Next, the WT $\alpha$-toxin and its variants (K110A, Y112A, and M113A mutant proteins) were expressed and purified as described by Qiu et al. (2012a), with the primers used for mutagenesis listed in Table 1. The fluorescence-quenching method was used to measure the binding constants $\left(K_{A}\right)$ of $\mathrm{AE}$ with the proteins. A $280-\mathrm{nm}$ excitation wavelength with a 5$\mathrm{nm}$ bandpass and a $345-\mathrm{nm}$ emission wavelength with a $10-\mathrm{nm}$ bandpass were used for the measurements. Details of performing the measurements were described previously (Bandyopadhyay et al., 2002; Jurasekova et al., 2009).

\section{Live/dead Assays and LDH Release}

A549 human lung epithelial cells (ATCC CCL-185) and mouse lung macrophages (MH-S, ATCC CRL-2019) were plated in 96well plates at a density of $2.0 \times 10^{4}$ cells/well and were cultured overnight. The cells were subsequently treated with $200 \mu \mathrm{l}$ of bacteria at an MOI of 500 with or without $\mathrm{AE}$ and then were incubated for $5 \mathrm{~h}$ at $37^{\circ} \mathrm{C}$. For the live/dead assays, the cells were stained using a live/dead (green/red) reagent (Invitrogen) and detected with a confocal laser scanning microscope (Nikon, Tokyo, Japan). For the LDH assay, the supernatants of the wells were assessed with a Cytotoxicity Detection Kit (LDH, Roche, Basel, Switzerland).

\section{Animal Infection}

Six-to-eight-week-old C57BL/6J female mice were purchased from Jilin University Experimental Animal Center. Animal experiments were approved by and conducted in accordance with the guidelines of the Animal Care and Use Committee of Jilin University. This study was approved by the Animal Welfare and Research Ethics Committee of Jilin University.

USA300 was cultured in TSB with shaking at $200 \mathrm{rpm}$ at $37^{\circ} \mathrm{C}$ to an $\mathrm{OD}_{600}$ of 0.8 , and the cells were then harvested by centrifugation for $10 \mathrm{~min}$ at 3,000 rpm. The bacteria were resuspended in physiological saline and quantified at $\mathrm{OD}_{600}$. The mice were anesthetized with ether and then were administered nasal drops with $20 \mu \mathrm{l}$ of suspended bacteria $\left(1 \times 10^{10}\right.$ CFUs/mL). The mice in the treatment group were subsequently injected with $100 \mathrm{mg} / \mathrm{kg} \mathrm{AE}$ by hypodermic injection $2 \mathrm{~h}$ after infection and were continually treated for $72 \mathrm{~h}$ at 8 -h intervals. The mice in the control group were treated with DMSO at the same dosage. Each experimental group in this study contained 10 mice.

TABLE 1 | Primers used in this study.

\begin{tabular}{ll}
\hline Primer & oligonucleotide primer sequence $\mathbf{( 5}^{\prime} \mathbf{~} \mathbf{3}^{\prime} \mathbf{)}$ \\
\hline WT-F & CGCGGATCCGCAGATTCTGATATTAATATTAAAC \\
WT-R & CCGCTCGAGTTATTGTCATTCTTCTITT \\
K110A-F & CCAAGAATTCGATTGATACAGCGGAGTATATGAGTACTITAAC \\
K110A-R & GTTAAAGTACTCATATACTCCGCTGTATCAATCGAATTCTTGG \\
T112A-F & GATTGATACAAAAGAGGCTATGAGTACTTAAC \\
T112A-R & GTTAAAGACTCATAGCCTCTITGTATCAATC \\
M113A-F & GATTGATACAAAAGAGTATGCGAGTACTTAACTTATGG \\
M113A-R & CCATAAGTTAAAGTACTCGCATACTCTITGTATCAATC
\end{tabular}

The mortality rate of the mice was determined daily. The mouse lungs were immersed in $4 \%$ formalin, embedded in paraffin, stained with hematoxylin and eosin, and visualized with a light microscope. For colony count analyses, the lungs were accurately weighed and ground in PBS containing 2\% Triton. Finally, the tissue homogenates were plated to quantify the colonization of USA300 in the lungs.

\section{Statistical Analysis}

The statistical analyses between the treated and control group were assessed using SPSS 13.0 software. The log-rank test and Student's $t$-tests were performed for the survival curves and others assays, respectively. ${ }^{*} P<0.05$ and ${ }^{* *} P<0.01$.

\section{RESULTS}

\section{AE has no Effect on S. aureus Growth}

The minimum inhibitory concentration (MIC) determination and growth curve assays were performed to determine the antibacterial activity of $\mathrm{AE}$ for $S$. aureus. The MIC of $\mathrm{AE}$ for the $S$. aureus strain USA300 was $>1,024 \mu \mathrm{g} / \mathrm{ml}$, suggesting that this compound, as a therapeutic agent, lacks antibacterial activity against USA300. Furthermore, USA300 grown with $2-16 \mu \mathrm{g} / \mathrm{ml}$ of $\mathrm{AE}$ showed no difference from the control group cultures without AE (Figure 1B).

\section{AE Inhibits the Hemolytic Activity of $\alpha$-toxin}

Then, the potential inhibitory effect of AE against $\alpha$-toxin activity was evaluated at the concentrations without antibacterial activity against $S$. aureus. The results of hemolysis assays showed that AE notably inhibited the hemolytic activity of the $S$. aureus supernatant in a dose-dependent manner when cocultured with bacteria. Consistent with previous studies, $80.97 \%$ of rabbit erythrocytes were lysed by $\alpha$-toxin in the control group without $\mathrm{AE}$, and the activity was significantly decreased (to $22.19 \%$ ) when cultured with $8 \mu \mathrm{g} / \mathrm{ml}$ of AE. Furthermore, only $4.00 \%$ of erythrocytes were lysed in the supernatants treated with $16 \mu \mathrm{g} / \mathrm{ml}$ of $\mathrm{AE}$ (Figure 1C). The $50 \%$ inhibitory concentration $\left(\mathrm{IC}_{50}\right)$ of AE determined to be $4.81 \mu \mathrm{g} / \mathrm{ml}$.

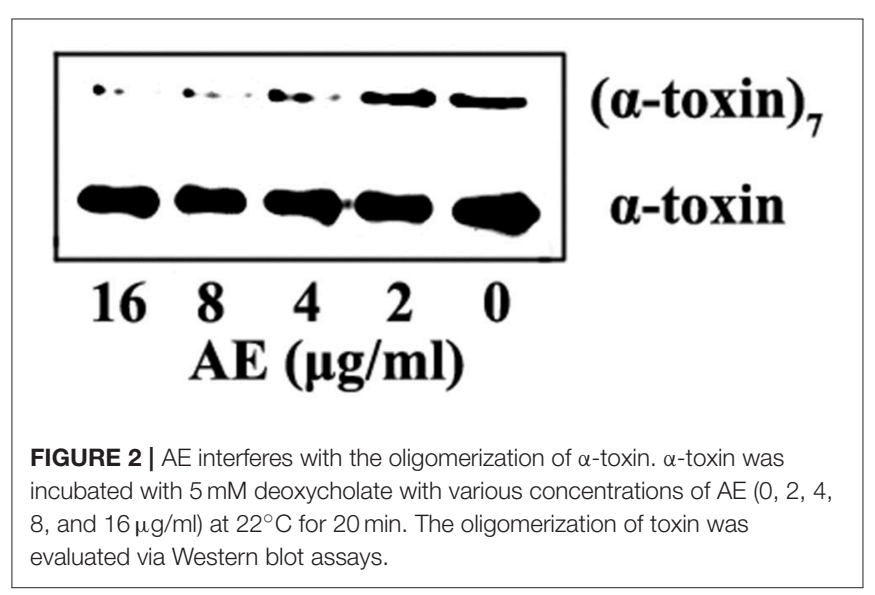


Western blot assays were used to further determine whether the decreased hemolytic activity was due to a reduction in $\alpha$ toxin. However, the secretion of $\alpha$-toxin was barely affected in the supernatants after treatment with various concentrations of $\mathrm{AE}$ (Figure 1D). Therefore, we hypothesized that AE may act on $\alpha$-toxin directly to inhibit its hemolytic activity. To validate this, we used recombinant purified $\alpha$-toxin in hemolysis assays. Consistent with the hemolysis described above, the hemolytic activity of purified $\alpha$-toxin was significantly inhibited in the presence of AE (Figure 1E). Taken together, our results suggested that $\mathrm{AE}$ inhibits the hemolytic activity of $\alpha$-toxin by direct interaction with the toxin.

\section{AE Inhibits the Oligomerization of $\alpha$-toxin}

Deoxycholate-induced oligomerization assays were used to determine whether the loss of hemolytic activity was due to the inhibition of oligomerization, which is critical for the hemolytic activity of $\alpha$-toxin. As expected, formation of the $\alpha$-toxin heptamer was significantly inhibited in the sample treated with $4 \mu \mathrm{g} / \mathrm{ml}$ of $\mathrm{AE}$, and the oligomerization was almost completely inhibited when $16 \mu \mathrm{g} / \mathrm{ml}$ of AE was added to the reaction system (Figure 2). Taken together, these results indicate that the loss of hemolytic activity may be due to AE-mediated inhibition of the oligomerization of $\alpha$-toxin.

\section{Molecular Dynamics Simulation for $\alpha$-toxin-AE}

Computational biology assays were further performed to characterize the detailed mechanisms of this inhibition. First, computational biology analyses were used to explore the potential binding mode of $\mathrm{AE}$ with $\alpha$-toxin in the active site. $\mathrm{AE}$ binds to $\alpha$-toxin, and the binding mode is shown in Figure $3 \mathbf{A}$. $\mathrm{AE}$ can bind to $\alpha$-toxin via hydrogen bonding and hydrophobic interactions. During the time course of the simulation, AE could localize to the "stem" region of $\alpha$-toxin (residue 100 to 150). In detail, the binding model of $\mathrm{AE}$ with $\alpha$-toxin revealed that the side chain of $\mathrm{AE}$ can form strong interactions with Lys110, Tyr112, and Met113. As shown in Figure 3B, the complex was found to reach equilibrium at $50 \mathrm{~ns}$ based on the analysis of the root-mean-square deviations (RMSD) of backbone $\mathrm{C}_{\alpha}$ atoms, which indicated that the complex system reached the equilibrium.

To explore the energy contributions from residues of the binding sites in the $\alpha$-toxin-AE complex, we calculated the energy

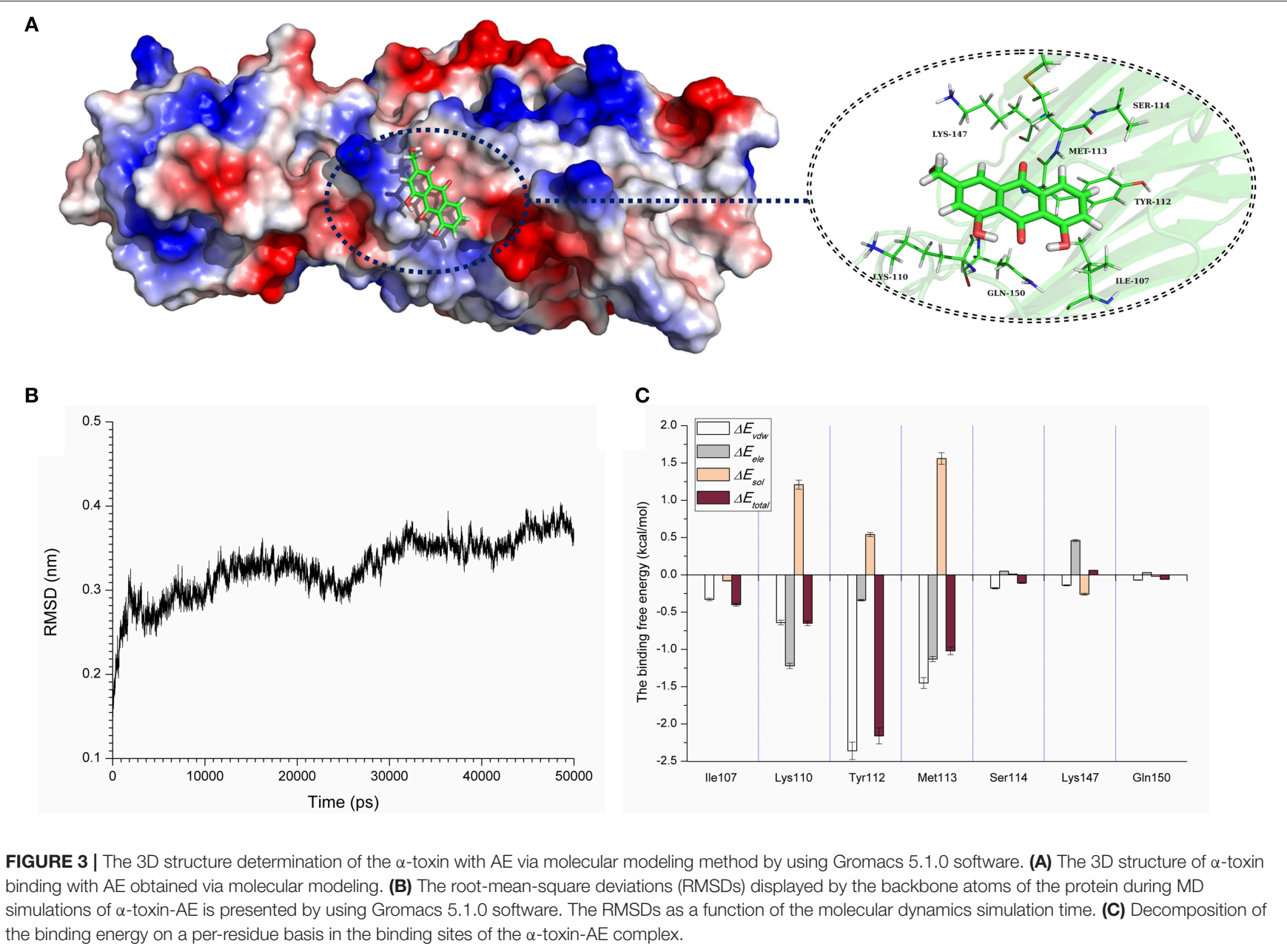


decomposition for the $\alpha$-toxin-AE complex system. As shown in Figure 3C, Tyr112 makes a strong contribution to the total binding energy, with an $\Delta E_{\text {total }} \leq-2.0 \mathrm{kcal} / \mathrm{mol}$. In addition, the Lys 110 and Met113 residues also have appreciable total binding energy contributions, with $\Delta E_{\text {total }}$ values of $\leq-0.5 \mathrm{kcal} / \mathrm{mol}$. The results suggest that these three residues are key residues for $\mathrm{AE}$.

To confirm these theoretical results, we calculated the total binding free energy for the $\alpha$-toxin-AE complex and their detailed energy contributions according to the MM-PBSA approach, which are summarized in Table 2. According to the calculation results, the binding free energy, $\Delta G_{b i n d}$, of the interaction between $\mathrm{AE}$ and the protein decreased in the following order: WT- $\alpha$-toxin $>$ mutants, indicating that the WT$\alpha$-toxin had the strongest AE-binding ability. By fluorescence spectroscopy quenching, we measured the $\Delta G_{b i n d}$ and the number of binding sites between $\mathrm{AE}$ and the three mutants, and these results were consistent with those obtained by computational methods (Table 2). Another hemolysis assay was used to determine the effect of AE on the hemolytic activity of the mutant proteins. As expected, the sensitivity of these mutants to $\mathrm{AE}$ was much lower than that of the WT protein, indicating that the mutations affected the binding of AE to $\alpha$-toxin (Figure 4).

Taken together, these results indicate that the molecular dynamics (MD) simulation of the $\alpha$-toxin-AE complex is reliable and that binding of the AE inhibitor at the active site (residues Lys110, Tyr112, and Met113) of $\alpha$-toxin leads to inhibition of its activity.

TABLE 2 | The binding free energy ( $\mathrm{kcal} / \mathrm{mol})$ of the WT- $\alpha$-toxin-AE, K110A-AE, Y112A-AE, and M113A-AE systems based on computational analysis and the values of the binding constants $\left(K_{A}\right)$ based on fluorescence spectroscopy quenching.

\begin{tabular}{lrrrr}
\hline WT- $\alpha$-toxin & K110A & Y112A & M113A \\
\hline Computational method & $-12.6 \pm 1.1$ & $-8.2 \pm 0.8$ & $-7.7 \pm 1.0$ & $-8.1 \pm 0.9$ \\
$K_{\mathrm{A}}\left(1 \times 10^{4}\right) \mathrm{L} \cdot \mathrm{mol}^{-1}$ & $8.9 \pm 0.8$ & $5.8 \pm 0.6$ & $6.1 \pm 0.9$ & $7.1 \pm 0.7$
\end{tabular}

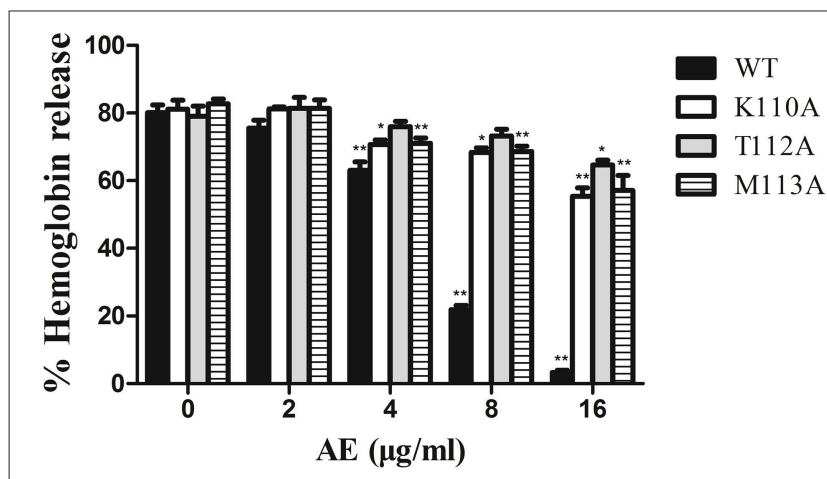

FIGURE 4 | The effect of AE on the hemolytic activity of $\alpha$-toxin and its three mutants. Recombinant $\alpha$-toxin was preincubated with various concentrations of $\mathrm{AE}$ and the hemolytic activity of toxin was determined using hemolysis assay as described in Figure 1E. Bars represent the mean values of the experiments ( ${ }^{*}$ indicates $P<0.05$ and ${ }^{* \star}$ indicates $P<0.01$ compared with the AE-free group; two-tailed Student's $t$-test).

\section{AE Protects A549 Cells From USA300-mediated Cell Injury}

Live/dead cell staining and lactate dehydrogenase (LDH) release assays were performed to assess the protective effects of $\mathrm{AE}$ on S. aureus-mediated A549 cell injury in the coculture system of $S$. aureus and cells with or without AE. After incubation for $5 \mathrm{~h}$, the cells were stained with live/dead (green/red) reagent (Figure 5A). As shown in Figure 5B, most of the cells were dead (red fluorescence) in the control group without AE. However, the cell injury was decreased with increasing concentrations of $\mathrm{AE}$, and almost no dead cells could be observed under a confocal laser scanning microscope when the concentration of $\mathrm{AE}$ reached $16 \mu \mathrm{g} / \mathrm{ml}$ (green fluorescence for live cells, Figure 5C). Furthermore, the cells treated with $16 \mu \mathrm{g} / \mathrm{ml}$ of AE only showed a normal cell morphology, with almost no dead cells (Figure 5D), suggesting that no toxicity could be observed at therapeutic concentrations.

$\mathrm{LDH}$ can be released into culture medium as an indicator of cell lysis. Thus, LDH release assays of A549 cells and mouse lung macrophages (MH-S) were used to quantify cell death. As shown in Figure 5E, $74.4 \%$ of A549 cells and $36.1 \%$ of $\mathrm{MH}-$ $\mathrm{S}$ cells were dead after incubation with USA300 for $5 \mathrm{~h}$ without AE. Significantly, when AE was added at $16 \mu \mathrm{g} / \mathrm{ml}$, the rate of cell death was decreased to 14.31 and $8.75 \%$, respectively (Figure 5E). Taken together, the results indicated that AE protected against cell injury mediated by $\alpha$-toxin in both A549 cells and MH-S cells in vitro.

\section{AE Protects Mice From S. aureus Pneumonia}

Following the determination of the protective effect of AE against $S$. aureus virulence in vitro, we further investigated whether this effect occurred in vivo by establishing a mouse model of $S$. aureus pneumonia. C57BL/6J mice were infected with USA300 as previously described (Qiu et al., 2012a,b) and treated with $\mathrm{AE}$ or DMSO for $72 \mathrm{~h}$. The mortality of infected mice that received $\mathrm{AE}$ was significantly decreased compared with that of mice injected with DMSO (Figure 6A). The lungs of the mice were pathologically analyzed. The redness of the lungs from the AE-treated group was much lighter than that of the control group when observed by the naked eye (Figure 6B), and the alveolar space was obliterated by inflammatory cell infiltrates in the control group as shown by microscopy (Figure 6C). Furthermore, we quantified the colonization of bacteria in the lungs. As shown in Figure 6D, the quantity of bacteria in lungs treated with $\mathrm{AE}$ was much lower than that of the control group. Taken together, our results indicated that $\mathrm{AE}$ is an effective candidate for protecting mice from $S$. aureus pneumonia.

\section{DISCUSSION}

Over the past 100 years, the application of antibiotics has saved countless lives, and scholars once believed that human beings could completely eradicate diseases caused by bacteria. However, the abuse of antibiotics has gradually increased resistance, which has finally led to the elimination of antibiotics after decades of 

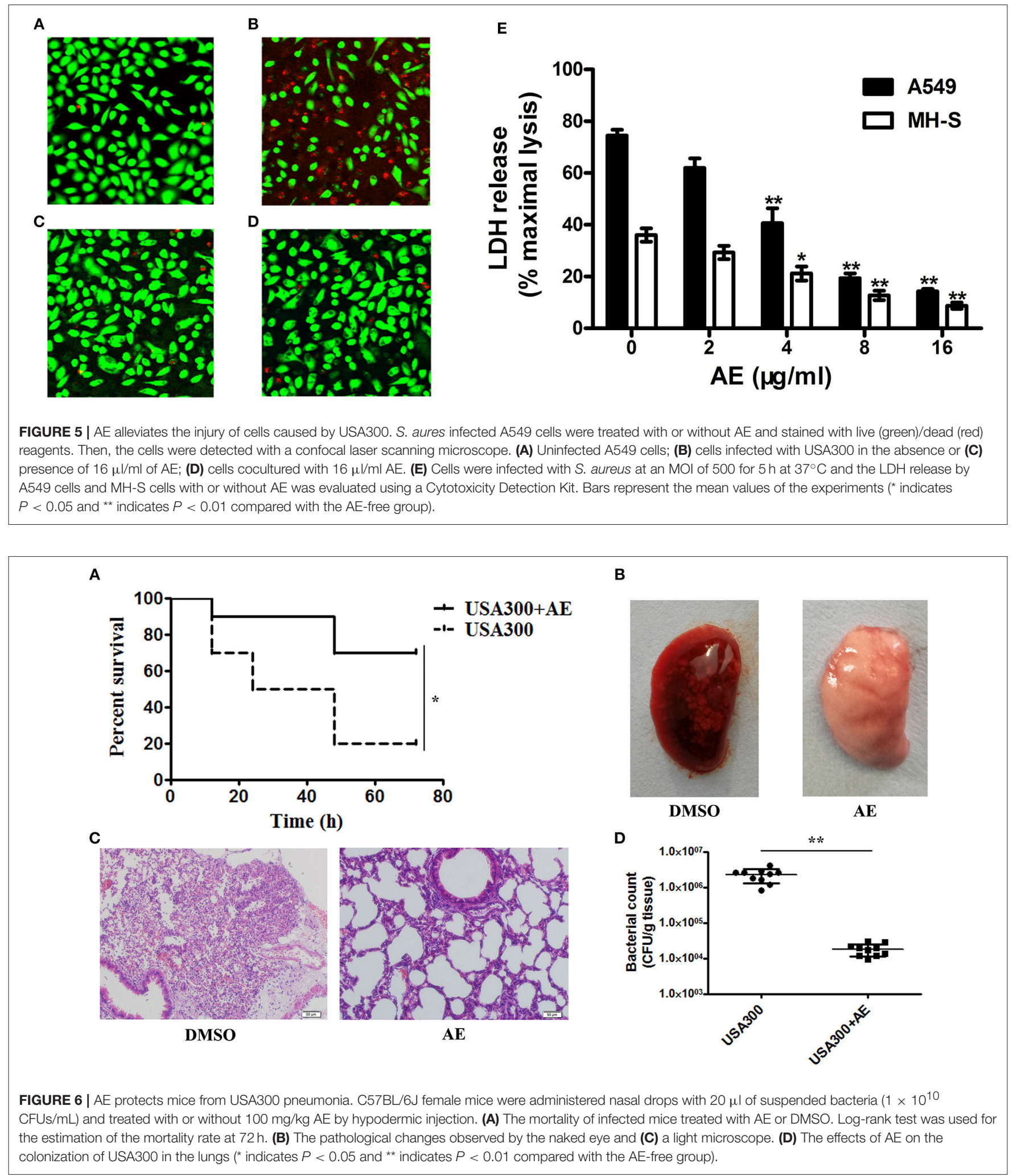

application and has resulted in many multidrug resistant strains that are insensitive to conventional antibacterial strategies, such as MRSA (Rasko and Sperandio, 2010). The use of antibiotics is typically related to the survival of bacteria and always leads to the selection of resistant strains that gradually become dominant and eventually results in resistant flora. Thus, it is possible that 
most antibiotics may eventually induce drug resistance due to the excellent ability of bacteria to adapt to the environment (Cegelski et al., 2008). Therefore, new treatment strategies are needed that are less effective at selecting for resistance. In this study, we observed that $\mathrm{AE}$ did not affect the viability of $S$. aureus at the concentrations tested $(2-16 \mu \mathrm{g} / \mathrm{ml})$. These results indicate that AE would not put selective pressure on $S$. aureus.

In recent years, many studies have suggested the targeting virulence factors as an alternative strategy and have shown some impressive results (Qiu et al., 2012a,b). For S. aureus infections, many virulence factors are employed during the invasion, and most of them, such as $\alpha$-toxin, are not correlated with bacterial survival. Based on previous studies, the destruction of the airblood barrier by $\alpha$-toxin is one of the most important factors in S. aureus pneumonia (McElroy et al., 1999; Xu et al., 2015). Accordingly, in this study, we attempted to treat $S$. aureusinduced pneumonia by targeting $\alpha$-toxin. We identified AE as a potential inhibitor of $\alpha$-toxin, as it directly interferes with the hemolytic activity of $\alpha$-toxin without affecting the production of $\alpha$-toxin by $S$. aureus or bacterial viability. By means of MD simulation, we found that $\mathrm{AE}$ could localize to the catalytic pocket of $\alpha$-toxin (residue 100-150), which is very close to the binding site of substrate, by directly engaging residues K110, T112, and M113. These interactions blocked the binding of $\alpha$ toxin and its substrate and finally led to a loss of biological activity of $\alpha$-toxin. Furthermore, we also demonstrated that $\mathrm{AE}$ had protective effects in a tissue infection model and animal model of $S$. aureus pneumonia. These results indicated that AE is a potential treatment for $S$. aureus infections, especially MRSA infections. In addition, our results confirmed the feasibility of the anti-virulence strategy.

Interestingly, Aloe vera, Rheum officinale, and semen cassiae torae, which are the major sources of $\mathrm{AE}$, have been used as traditional Chinese medicines in the treatment of lung infections for thousands of years. Although this study cannot show that the effect of $\mathrm{AE}$ on $\alpha$-toxin is the major mechanism of these medicines on lung infections, it provides support for the theory of traditional Chinese medicine at the molecular level.

\section{REFERENCES}

Bandyopadhyay, S., Ren, H., Wang, C.S., and Allison, W.S. (2002). The (alpha $\mathrm{F}(357) \mathrm{C})(3)$ (beta $\mathrm{R}(372) \mathrm{C})(3)$ gamma subcomplex of the $\mathrm{F}(1)$-ATPase from the thermophilic Bacillus PS3 has altered ATPase activity after cross-linking alpha and beta subunits at noncatalytic site interfaces. Biochemistry 41, 3226-3234. doi: 10.1021/bi01 20291

Cegelski, L., Marshall, G.R., Eldridge, G.R., and Hultgren, S.J. (2008). The biology and future prospects of antivirulence therapies. Nat. Rev. Microbiol. 6, 17-27. doi: $10.1038 /$ nrmicrol 1818

Chastre, J., Blasi, F., Masterton, R.G., Rello, J., Torres, A., and Welte, T. (2014). European perspective and update on the management of nosocomial pneumonia due to methicillin-resistant Staphylococcus aureus after more than 10 years of experience with linezolid. Clin. Microbiol. Infect. 20 (Suppl. 4), 19-36. doi: 10.1111/1469-0691.12450

Chen, C.J., and Huang, Y.C. (2014). New epidemiology of Staphylococcus aureus infection in Asia. Clin. Microbiol. Infect. 20, 605-623. doi: 10.1111/1469-0691.12705
Previous studies have reported that $\alpha$-toxin can also damage the membranes of $\mathrm{B}$ cells and $\mathrm{T}$ cells, which may lead to an attenuated immune response and create a stable and lasting bacterial colonization environment (Nygaard et al., 2012). As reported in several studies, subinhibitory concentrations of beta-lactam antibiotics may increase $\alpha$-toxin expression, indicating that infections may be uncontrollable when treated with beta-lactams (Ohlsen et al., 1998). Therefore, targeting $\alpha$-toxin by $\mathrm{AE}$ may support the therapeutic effects of immune cells and antibiotics, but this strategy requires further confirmation. Finally, our results indicated that $\mathrm{AE}$ is a promising candidate for $S$. aureus infection by targeting $\alpha$-toxin.

\section{DATA AVAILABILITY}

The raw data supporting the conclusions of this manuscript will be made available by the authors, without undue reservation, to any qualified researcher.

\section{ETHICS STATEMENT}

Six-to-eight-week-old C57BL/6J female mice were purchased from Jilin University Experimental Animal Center and were handled according to the standards approved by the Animal Welfare and Research Ethics Committee of Jilin University.

\section{AUTHOR CONTRIBUTIONS}

ZT, JW, and LJ conceived and designed the experiments. LJ, TY, and ZS performed the experiments. LJ analyzed the data. ZT and JW wrote the paper.

\section{FUNDING}

This work was supported by the National Key Technology R\&D Program (No. 2016YFD05013) and the National Natural Science Foundation of China (grant 31602109).

Commun, E. (2011). European Centre for Disease Prevention and Control publishes Annual epidemiological report 2011. Eurosurveillance 16:17.

Del Giudice, P., Bes, M., Hubiche, T., Blanc, V., Roudière, L., Lina, G., et al. (2011). Panton-Valentine leukocidin-positive Staphylococcus aureus strains are associated with follicular skin infections. Dermatology 222, 167-170. doi: 10.1159/000324044

Dong, J., Qiu, J., Zhang, Y., Lu, C., Dai, X., Wang, J., et al. (2013). Oroxylin A inhibits hemolysis via hindering the self-assembly of alphahemolysin heptameric transmembrane pore. PLoS Comput. Biol. 9:e1002869. doi: 10.1371/journal.pcbi.1002869

Dutta, A., Bandyopadhyay, S., Mandal, C., and Chatterjee, M. (2007). Aloe vera leaf exudate induces a caspase-independent cell death in Leishmania donovani promastigotes. J. Med. Microbiol. 56 (Pt 5), 629-636. doi: 10.1099/jmm.0.47039-0

Eshun, K., and He, Q. (2004). Aloe vera: a valuable ingredient for the food, pharmaceutical and cosmetic industries-a review. Crit. Rev. Food Sci. Nutr. 44, 91-96. doi: 10.1080/10408690490424694

Gillet, Y., Issartel, B., Vanhems, P., Fournet, J.C., Lina, G., Bes, M., et al. (2002). Association between Staphylococcus aureus strains carrying 
gene for Panton-Valentine leukocidin and highly lethal necrotising pneumonia in young immunocompetent patients. Lancet 359, 753-759. doi: 10.1016/S0140-6736(02)07877-7

Gouaux, E. (1998). alpha-Hemolysin from Staphylococcus aureus: an archetype of beta-barrel, channel-forming toxins. J. Struct. Biol. 121, 110-122. doi: 10.1006/jsbi.1998.3959

Gouaux, J.E., Braha, O., Hobaugh, M.R., Song, L., Cheley, S., Shustak, C., et al. (1994). Subunit stoichiometry of staphylococcal alpha-hemolysin in crystals and on membranes: a heptameric transmembrane pore. Proc. Natl. Acad. Sci. U.S.A. 91, 12828-12831.

Hu, R., Barbault, F., Maurel, F., Delamar, M., and Zhang, R. (2010). Molecular dynamics simulations of 2-amino-6-arylsulphonylbenzonitriles analogues as HIV inhibitors: interaction modes and binding free energies. Chem. Biol. Drug Des. 76, 518-526. doi: 10.1111/j.1747-0285.2010.01028.x

Jurasekova, Z., Marconi, G., Sanchez-Cortes, S., and Torreggiani, A. (2009). Spectroscopic and molecular modeling studies on the binding of the flavonoid luteolin and human serum albumin. Biopolymers 91, 917-927. doi: 10.1002/bip. 21278

Kuo, P. L., Lin, T. C., and Lin, C. C. (2002). The antiproliferative activity of aloe-emodin is through p53-dependent and p21-dependent apoptotic pathway in human hepatoma cell lines. Life Sci. 71, 1879-1892. doi: 10.1016/s0024-3205(02)01900-8

Lee, H.Z. (2001). Protein kinase C involvement in aloe-emodin- and emodininduced apoptosis in lung carcinoma cell. Br. J. Pharmacol. 134, 1093-1103. doi: 10.1038/sj.bjp.0704342

Li, H. T., Zhang, T. T., Huang, J., Zhou, Y. Q., Zhu, J. X., and Wu, B. Q. (2011). Factors associated with the outcome of life-threatening necrotizing pneumonia due to community-acquired Staphylococcus aureus in adult and adolescent patients. Respiration 81, 448-460. doi: 10.1159/0003 19557

McElroy, M. C., Harty, H. R., Hosford, G. E., Boylan, G. M., Pittet, J. F., and Foster, T. J. (1999). Alpha-toxin damages the air-blood barrier of the lung in a rat model of Staphylococcus aureus-induced pneumonia. Infect. Immun. 67, 5541-5544.

Mendes, R. E., Mendoza, M., Banga Singh, K. K., Castanheira, M., Bell, J. M., Turnidge, J. D., et al. (2013). Regional resistance surveillance program results for 12 Asia-Pacific nations (2011). Antimicrob. Agents Chemother. 57, 5721-5726. doi: 10.1128/AAC.01121-13

Morris, G. M., Huey, R., Lindstrom, W., Sanner, M. F., Belew, R. K., Goodsell, D. S., et al. (2009). AutoDock4 and AutoDockTools4: automated docking with selective receptor flexibility. J. Comput. Chem. 30, 2785-2791. doi: $10.1002 /$ jcc. 21256

Nguyen, V. T., and Kamio, Y. (2004). Cooperative assembly of beta-barrel poreforming toxins. J. Biochem. 136, 563-567. doi: 10.1093/jb/mvh160

Niu, X., Qiu, J., Wang, X., Gao, X., Dong, J., Wang, J., et al. (2013). Molecular insight into the inhibition mechanism of cyrtominetin to alphahemolysin by molecular dynamics simulation. Eur. J. Med. Chem. 62, 320-328. doi: 10.1016/j.ejmech.2013.01.008

Nygaard, T. K., Pallister, K. B., DuMont, A. L., DeWald, M., Watkins, R. L., Pallister, E. Q., et al. (2012). Alpha-toxin induces programmed cell death of human T cells, B cells, and monocytes during USA300 infection. PLoS ONE 7:e36532. doi: 10.1371/journal.pone.0036532

Ohlsen, K., Ziebuhr, W., Koller, K.P., Hell, W., Wichelhaus, T.A., and Hacker, J. (1998). Effects of subinhibitory concentrations of antibiotics on alpha-toxin (hla) gene expression of methicillin-sensitive and methicillinresistant Staphylococcus aureus isolates. Antimicrob. Agents Chemother. 42, 2817-2823.

Parvizi, J., Pawasarat, I.M., Azzam, K.A., Joshi, A., Hansen, E.N., and Bozic, K.J. (2010). Periprosthetic joint infection: the economic impact of methicillin-resistant infections. J. Arthroplasty 25 (Suppl. 6), 103-107. doi: 10.1016/j.arth.2010.04.011

Pecere, T., Gazzola, M.V., Mucignat, C., Parolin, C., Vecchia, F.D., Cavaggioni, A., et al. (2000). Aloe-emodin is a new type of anticancer agent with selective activity against neuroectodermal tumors. Cancer Res. 60, 2800-2804.

Qiu, J., Niu, X., Dong, J., Wang, D., Wang, J., Li, H., et al. (2012a). Baicalin protects mice from Staphylococcus aureus pneumonia via inhibition of the cytolytic activity of alpha-hemolysin. J. Infect. Dis. 206, 292-301. doi: 10.1093/infdis/jis336

Qiu, J., Niu, X., Wang, J., Xing, Y., Leng, B., Dong, J., et al. (2012b). Capsaicin protects mice from community-associated methicillin-resistant Staphylococcus aureus pneumonia. PLoS ONE 7:e33032. doi: 10.1371/journal.pone.0033032

Rasko, D.A., and Sperandio, V. (2010). Anti-virulence strategies to combat bacteria-mediated disease. Nat. Rev. Drug Discov. 9, 117-128. doi: $10.1038 / \mathrm{nrd} 3013$

Song, L., Hobaugh, M.R., Shustak, C., Cheley, S., Bayley, H., and Gouaux, J.E. (1996). Structure of staphylococcal alpha-hemolysin, a heptameric transmembrane pore. Science 274, 1859-1866.

Song, X., Perencevich, E., Campos, J., Short, B.L., and Singh, N. (2010). Clinical and economic impact of methicillin-resistant Staphylococcus aureus colonization or infection on neonates in intensive care units. Infect. Control Hosp. Epidemiol. 31, 177-182. doi: 10.1086/649797

Vandenesch, F., Lina, G., and Henry, T. (2012). Staphylococcus aureus hemolysins, bi-component leukocidins, and cytolytic peptides: a redundant arsenal of membrane-damaging virulence factors? Front. Cell. Infect. Microbiol. 2:12. doi: 10.3389/Fcimb.2012.00012

Wang, J., Zhou, X., Li, W., Deng, X., Deng, Y., and Niu, X. (2016). Curcumin protects mice from Staphylococcus aureus pneumonia by interfering with the self-assembly process of alpha-hemolysin. Sci. Rep. 6:28254. doi: $10.1038 /$ srep 28254

Wunderink, R. G., Rello, J., Cammarata, S. K., Croos-Dabrera, R. V., and Kollef, M. H. (2003). Linezolid vs vancomycin: analysis of two double-blind studies of patients with methicillin-resistant Staphylococcus aureus nosocomial pneumonia. Chest 124, 1789-1797.

Xu, F., Diao, R., Liu, J., Kang, Y., Wang, X., and Shi, L. (2015). Curcumin attenuates Staphylococcus aureus-induced acute lung injury. Clin. Respir. J. 9, 87-97. doi: $10.1111 /$ crj.12113

Conflict of Interest Statement: The authors declare that the research was conducted in the absence of any commercial or financial relationships that could be construed as a potential conflict of interest.

Copyright (c) 2019 Jiang, Yi, Shen, Teng and Wang. This is an open-access article distributed under the terms of the Creative Commons Attribution License (CC BY). The use, distribution or reproduction in other forums is permitted, provided the original author(s) and the copyright owner(s) are credited and that the original publication in this journal is cited, in accordance with accepted academic practice. No use, distribution or reproduction is permitted which does not comply with these terms. 